\title{
An Open Trial of In-Home CBT for Depressed Mothers in Home Visitation
}

\author{
Robert T. Ammerman, \\ Department of Pediatrics, Cincinnati Children's Hospital Medical Center, University of Cincinnati \\ College of Medicine, 3333 Burnet Avenue, ML 3015, Cincinnati, OH 45229, USA \\ Frank W. Putnam, \\ Department of Pediatrics, Cincinnati Children's Hospital Medical Center, University of Cincinnati \\ College of Medicine, 3333 Burnet Avenue, ML 3008, Cincinnati, OH 45229, USA \\ Jack Stevens, \\ Department of Pediatrics, Nationwide Children's Hospital, The Ohio State University School of \\ Medicine, Columbus, $\mathrm{OH}$, USA
}

\begin{abstract}
Nicole R. Bosse,
Department of Pediatrics, Cincinnati Children's Hospital Medical Center, University of Cincinnati College of Medicine, 3333 Burnet Avenue, ML 3015, Cincinnati, OH 45229, USA
\end{abstract}

Jodie A. Short,

Department of Pediatrics, Cincinnati Children's Hospital Medical Center, University of Cincinnati College of Medicine, 3333 Burnet Avenue, ML 3005, Cincinnati, OH 45229, USA

\section{Amy L. Bodley, and \\ Department of Pediatrics, Cincinnati Children's Hospital Medical Center, University of Cincinnati College of Medicine, 3333 Burnet Avenue, ML 3015, Cincinnati, OH 45229, USA \\ Judith B. Van Ginkel \\ Department of Pediatrics, Cincinnati Children's Hospital Medical Center, University of Cincinnati College of Medicine, 3333 Burnet Avenue, ML 3005, Cincinnati, OH 45229, USA}

Robert T. Ammerman: robert.ammerman@cchmc.org; Frank W. Putnam: frank.putnam@cchmc.org; Jack Stevens: jack.stevens@nationwidechildrens.org; Nicole R. Bosse: nicole.bosse@cchmc.org; Jodie A. Short:

jodie.short@cchmc.org; Amy L. Bodley: amy.bodley@cchmc.org; Judith B. Van Ginkel: judith.vanginkel@cchmc.org

\section{Abstract}

Research has demonstrated that low income mothers participating in home visitation programs have high rates of depression. This study used an open trial design to evaluate In-Home Cognitive Behavioral Therapy (IH-CBT), an evidence-based treatment for depression that is delivered in the home setting and has been adapted to address the needs of low income mothers participating in home visitation. 64 depressed mothers recruited from a home visitation program and who had completed IH-CBT were compared to 241 mothers from the same setting who met identical screening criteria at enrollment but did not receive the treatment. In addition, pre- and posttreatment measures of depression and related clinical features were contrasted in the 64 mothers receiving IH-CBT. There was a significantly greater reduction in depressive symptoms in the IHCBT group relative to their counterparts who did not receive the treatment. Results from pre-post comparisons showed that treated mothers had decreased diagnosis of major depression, lower reported stress, increased coping and social support, and increased positive views of motherhood

(C) Springer Science+Business Media, LLC 2010

Correspondence to: Robert T. Ammerman, robert . ammerman@ cchmc org. 
at post-treatment. Findings suggest that IH-CBT is a promising approach to addressing maternal depression in the context of home visitation and warrants further study. Public health implications for home visiting programs are discussed.

\section{Keywords}

Home visitation; Maternal depression; Cognitive behavioral therapy; Child abuse prevention

\section{Introduction}

Maternal depression has profound negative effects on parenting and child development [1]. Research further indicates that depressed mothers generally, and low income mothers in particular, rarely receive evidence-based treatments [2]. Home visitation, a child abuse prevention approach for high risk mothers and their children, is a potentially important setting in which depressed mothers can be identified and treated. Home visitation seeks to promote optimal child development through the strengthening of protective factors and prevention of negative outcomes, including academic underachievement, psychological maladjustment, and antisocial behavior [3]. Home visitation programs typically enroll families based on maternal sociodemographic risk factors (e.g., low income, young age) that are associated with elevated risk for poor parenting outcomes. In home visitation, a home visitor provides psychoeducational training and case management services to mothers and children. Research suggests that, when implemented in a focused way and with fidelity, home visitation programs can yield positive outcomes in child health and development and maternal life course in high risk families $[4,5]$.

There are currently up to 500,000 children receiving home visitation services in the USA [6] reflecting a substantial public health investment. Such programs are the earliest portals through which sizable numbers of high risk mothers come to the attention of service providers. Research has consistently demonstrated that home visitation includes a large population of depressed mothers. Indeed, mothers in home visitation typically have characteristics that are risk factors for the development of depression including poverty, social isolation, and trauma history [7]. For example, Ammerman et al. [8] found that, in 806 first-time, at-risk mothers enrolled in a regional home visitation program, $45.3 \%$ of mothers obtained a score of $\geq 14$ on the Beck Depression Inventory-II [9] in the first year of service. Similarly high rates have been found in studies of Early Head Start [10], Hawaii Healthy Start [11] and Healthy Families New York [12]. Although clinically elevated depressive symptoms are relatively common in home visitation programs, the vast majority of studies have found no benefits of home visitation relative to control conditions for maternal depression during the interval of service delivery [8].

In-Home Cognitive Behavioral Therapy (IH-CBT [13]) was developed to address the shortcomings in service availability for at-risk mothers in home visitation. IH-CBT is an adapted treatment in which an evidence-based approach (i.e., CBT) has been modified and tailored to address depression in high risk, first-time mothers who are participating in home visitation. IH-CBT uses the core features of CBT [14] and adds innovative delivery, tools, formats, and approaches to maximize effectiveness and integration with public health services. IH-CBT is provided in the home setting, thereby removing a significant barrier to obtaining mental health care. In addition, it seeks to leverage ongoing home visitation to improve both depression outcomes and parenting and child development [15]. IH-CBT is manualized thereby facilitating dissemination to other public health settings. It contains adaptations to address concurrent clinical needs (including history of trauma, intimate partner violence, poverty, and family instability) and cultural features [16] that require 
focused attention in order to maximize recovery from depression. A brief report of 26 mothers who received IH-CBT provided preliminary evidence supporting the effectiveness of the treatment [13]. The current study augments this sample with an additional 38 mothers and compares them to an untreated sample of depressed mothers in home visitation, and provides a more in-depth examination of the impact of IH-CBT on maternal functioning.

\section{Method}

\section{Participants}

The study consisted of two groups of first-time, high risk mothers aged $\geq 18$ years of age who were recruited from a community-based home visitation program. All mothers were enrolled in home visitation between 2002 and 2006 and met home visitation program enrollment criteria of having at least one of three risk characteristics: unmarried; low income ( $<300 \%$ of poverty, or Medicaid recipient); and inadequate prenatal care (late, insufficient, or no pre-natal care). The first group (Treatment Group) consisted of 64 mothers who were identified through screening based on an elevated score ( 20 ) on the Beck Depression Inventory-II [9] and subsequently met criteria for Major Depressive Disorder, and received and completed In-Home Cognitive Behavioral Therapy (IH-CBT) during the first 2 years of the child's life. Mothers in this group were assessed for depression and other areas of adjustment at pre- and post-treatment. The second group (Comparison Group) consisted of 241 mothers who were enrolled prior to the implementation of IH-CBT, and sequentially identified at enrollment as having scores $\geq 20$ on the BDI-II. Mothers in this group were assessed for depression at enrollment and 9 months later as part of pre-determined clinical practice for regular screening in the home visitation program. Home visitors documented that $19.9 \%$ of Comparison Group mothers received mental health treatment in the community during the enrollment-9 month interval.

Table 1 shows the demographic characteristics of the samples. Both groups were young, primarily unmarried, and low income. Group differences were found on several variables. Relative to the Comparison Group, those in the Treatment Group were older, more likely to be African American, achieved a higher level of education, and had higher scores at first administration of the BDI-II. Each of these was considered as covariates in subsequent comparisons.

\section{Recruitment Setting}

Mothers were enrolled in Every Child Succeeds (ECS), a regional home visitation program serving seven counties in Southwest Ohio and Northern Kentucky. ECS utilizes two national models of home visitation: Nurse-Family Partnership (NFP [17]) and Healthy Families America (HFA [18]). Mothers were enrolled prior to 28 weeks gestation in NFP, and from 20 weeks gestation through the child reaching 3 months of age for HFA as per model parameters. Mothers were referred from prenatal clinics, hospitals, social service agencies, and community physicians. In the Treatment Group, $85.9 \%$ of mothers received the HFA model vs. $14.1 \%$ in NFP, and in the Comparison Group $82.2 \%$ received the HFA model vs. $17.8 \%$ in NFP $\left(\chi^{2}(1)=0.51, P>.05\right)$.

\section{Procedures}

Participants were recruited and data were collected as part of ongoing home visitation and implementation of a clinical service for depressed mothers. Mothers in the Treatment Group were identified using the Beck Depression Inventory-II (BDI-II [9]). Specifically, the BDI-II was administered to all mothers at three scheduled time points: enrollment, and at 9 and 18 months post-enrollment. Eligibility was determined in a two step process. First, mothers obtaining a BDI-II score of $\geq 20$ were referred for an eligibility assessment. This cutoff was 
selected given the overall higher scores observed among low income mothers in home visitation who typically live in high stress and low resource environments [7] and they were more likely to meet diagnostic criteria for Major Depressive Disorder. [19] In the second step, a semi-structured diagnostic interview (PRIME-MD [20]) was administered 2 weeks later to confirm presence of MDD. Exclusionary criteria consisted of mental retardation, neurological disease, substance dependence, currently receiving psychotherapy, and/or acute suicidality or homicidality. Mothers were permitted to take a psychotropic medication provided they had been on a stable dose for $\geq 2$ months prior to BDI-II administration; 6 mothers $(9.4 \%)$ were taking medications at pre-treatment assessment. Post-treatment assessments were conducted upon completion of treatment, about $4 \frac{1}{2}$ months after the pretreatment assessment.

There were 173 mothers initially assessed for eligibility for treatment. Of those assessed, 138 met inclusion and exclusion criteria, and 35 were found to be ineligible (yielding a rate of $79.8 \%$; reasons for ineligibility were: did not meet criteria for $\mathrm{MDD}=9$, received exclusionary psychiatric diagnosis $=14$, acute suicidality $=4$, mental retardation $=4$, currently receiving psychotherapy $=4)$. Comparisons between eligible and ineligible mothers on demographics and service variables revealed no significant differences $(P>.05)$ on maternal age, maternal race, marital status, maternal education, family income, home visitation model, or baseline BDI-II scores. Of eligible mothers, 118 started treatment (had at least one session) and $20 \mathrm{did}$ not (yielding a rate of 85.6\%). Reasons for not starting treatment included mothers changing their minds about participation or multiple failed attempts to engage mothers. Comparisons between those who started and did not start treatment on demographics and service variables revealed no significant differences $(P>$. 05 ) on maternal age, maternal race, marital status, maternal education, family income, home visitation model, or baseline BDI-II scores. Of the 118 mothers who started treatment 64 completed it (yielding a rate of 54.2\%). An intent to treat strategy was not used given the service environment in which the treatment was implemented. Comparisons between those who completed and did not complete treatment on demographics and service variables revealed no significant differences $(P>.05)$ on maternal race, marital status, family income, and home visitation model, or baseline BDI-II scores. Relative to non-completers, those who completed treatment were older $(\mathrm{M}=22.6(\mathrm{SD}=5.0)$ vs. $\mathrm{M}=20.7(\mathrm{SD}=2.7), t(1,117)=$ $2.5, P<.05)$ and had higher levels of education $(71.9 \%$ vs. $35.2 \%$ had at least a high school degree or GED, $\left.\chi^{2}(1)=23.7, P<.01\right)$.

Mothers in the Comparison Group were identified sequentially based on having obtained a BDI-II score of $\geq 20$ at enrollment in home visitation thereby ensuring equivalence on this screening criterion with the Treatment Sample. They were followed over the first 9 months of home visitation at which point the BDI-II was re-administered. All procedures and consent were approved by the Institutional Review Board at Cincinnati Children's Hospital Medical Center.

\section{Treatment Description}

In-Home Cognitive Behavioral Therapy (IH-CBT) is grounded in the core principles and established procedures of CBT for depression originally articulated by Beck and colleagues [21]. IH-CBT was delivered in the home by a licensed master's level social worker.

Treatment consisted of 15 sessions that were scheduled weekly and lasted 60 min plus a booster session 1 month post-treatment.

IH-CBT preserves the core features of CBT that are essential to its effectiveness [22] while modifying elements of treatment content and delivery in order to enhance feasibility, engagement, and impact. In IH-CBT, adaptations were made to address setting, population, and context. These adaptations were made based upon a review of the literature, consultation 
with home visitors, and input from mothers in home visitation. First, IH-CBT was delivered in the home environment. Creative solutions and accommodations were made to ensure treatment delivery in home environments where privacy was sometimes difficult to ensure, the child was present, and unexpected interruptions occurred. However, providing treatment in the home offered advantages in that many of the clinical issues that were addressed in treatment occurred in the home setting, and the therapist was able to observe elements of the home that may have been contributory to psychopathology. The second adaptation involved the population of young, low income, first-time mothers who were socially isolated.

Treatment content focused on issues relevant to this population, including transition to adulthood, parenting efficacy, relationship maladjustment, and trauma history. The third adaptation sought to leverage participation in home visitation in order to optimize outcomes through close collaboration with home visitors. Collaboration occurred through frequent written communication between therapist and home visitor utilizing a web-based clinical documentation system, and telephone contact as needed. In addition, the home visitor attended the 15th session with the mother and therapist. Weekly supervision was provided by doctoral level clinicians who monitored fidelity to CBT and to the adaptations comprising the IH-CBT approach.

\section{Measures}

The Beck Depression Inventory-II (BDI-II [9]) is one of the most widely used screens for depression. It has been extensively used in clinical trials of treatments of depression and has been used with postpartum women. Its strong psychometric properties are well-documented [9]. In this study, the BDI-II was analyzed in three ways consistent with practices in treatment outcome research in depression [23]: (1) total score, (2) proportion obtaining a $50 \%$ reduction in symptoms from first to second administrations reflecting clinically significant improvement, and [3] proportion obtaining a score of $\$ 8$ indicating asymptomatic status. For the Treatment Group, the BDI-II was administered at the initial screening and at post-treatment. For the Comparison Group, it was administered at enrollment and 9-month post-enrollment.

The PRIME-MD [20] was used to diagnose Major Depressive Disorder. The PRIME-MD is a semi-structured psychiatric interview designed for use in clinical settings that uses DSM criteria. It is divided into five modules reflecting common psychiatric disorders encountered in clinical practice: mood, anxiety, alcohol, eating, and somatoform. Psychometric studies have documented the reliability of the measure and demonstrated validity through concordance with other diagnostic procedures [20]. The PRIME-MD was administered by an independent assessor and was used to diagnose depression at the pre-treatment and posttreatment assessments for the Treatment Group only.

The Brief Patient Health Questionnaire (BPHQ [24]) provided a self-report of depressive symptoms and associated clinical features. The BPHQ is designed to accompany the PRIME-MD and it consists of four parts. The first part, referred to as the PHQ-9, is comprised of 9 items measuring depressive symptoms. Each item is endorsed based on a 4point scale (not at all, several days, more than half the days, nearly every day) indicating how often in the past 2 weeks the symptom was experienced yielding a total score. The second part consists of five items reflecting symptoms of panic disorder. The first item screens for likelihood of panic disorder with a yes/no response ("In the last 4 weeks, have you had an anxiety attack suddenly feeling fear or panic?"). Endorsement of "yes" leads to four additional items answered in a yes/no format reflecting frequency and severity of panic symptoms. Endorsing all four items as "yes" indicates likelihood of meeting diagnostic criteria for panic disorder. The third part consists of a single item reflecting functional impairment ("how difficult have these problems made it to do your work, take care of things at home or get along with other people?") that is endorsed using a 4-point scale reflecting 
degree of difficulty (not at all difficult, somewhat difficult, very difficult, extremely difficult). The fourth part is comprised of ten psychosocial stressors. The BPHQ also contains an item screening for physical or sexual violence in the past year $(23.4 \%$ of the Treatment Group mothers endorsed this item).

The Maternal Attitudes Questionnaire (MAQ [25]) was used to measure maternal views of their children. The MAQ is a 14-item measure of expectations of and attitudes towards motherhood, developed originally for use with depressed mothers. Each item is endorsed using a 4-point Likert scale reflecting agreement with each item. High scores reflect increased dissatisfaction and negative attitudes towards motherhood. Warner et al. [25] reported an association between the MAQ and depression, and an internal reliability coefficient of .84, findings that were also reported by Church et al. [26]. The MAQ was administered at the pre-treatment assessment and at post- treatment for the Treatment Group only.

\section{Results \\ Group Comparisons}

Treatment and Comparison Groups were contrasted on the total scores of the BDI-II at first (pre-treatment and enrollment) and second (post-treatment and 9 month) administrations using a 3-way ANCOVA with repeated measures. Group (Treatment vs. Comparison), race (Caucasian vs. African American), and education (no high school degree vs. high school degree or higher) were factors. Years of education, maternal age, and first administration of the BDI-II were covariates. A significant main effect was obtained for race $(F(1,275)=4.5$, $P<.05)$, but there were no interaction effects for Group $\mathrm{x}$ race or Group $\mathrm{x}$ education $(P>$. $05)$. A significant race $x$ time effect revealed that African Americans $(M=28.0(S D=6.6)$ to $18.0(\mathrm{SD}=10.8)$ ) had higher BDI-II scores relative to Caucasians $(\mathrm{M}=28.8(\mathrm{SD}=7.7)$ to $15.6(\mathrm{SD}=11.0))$ at time 2 . Results revealed a statistically significant effect for Group $\mathrm{x}$ time $(F(1,281)=12.2, P<.01)$. Although both groups showed a reduction in depressive symptoms from times 1 to 2, the decrease was significantly larger for the Treatment Group $(\mathrm{M}=31.7(\mathrm{SD}=7.5)$ to $13.3(\mathrm{SD}=9.2))$ vs. Comparison Group $(\mathrm{M}=27.7(\mathrm{SD}=7.0)$ to $17.1(\mathrm{SD}=11.4))$.

In terms of time 2 endpoint reflections of depressive symptoms, mothers in the Treatment Group were more likely to have had a 50\% reduction in BDI-II scores relative to those in the Comparison Group $\left(65.6 \%\right.$ vs. $\left.34.4 \%, \chi^{2}(1)=11.4, P<.01\right)$ and more often obtained a BDI-II score $\leq 8$ reflecting asymptomatic status $\left(39.1 \%\right.$ vs. $\left.24.1 \%, \chi^{2}(1)=5.7, P<.05\right)$. Within the Comparison Group, there was no significant difference between mothers who received or did not receive mental health treatment during the 9 month interval $(F(1,237)=$ $0.84, P>.05)$.

\section{Pre- and Post-Treatment Contrasts in Treatment Group}

In the Treatment Group, at the end of treatment $46.9 \%$ of mothers met criteria for partial (MDD resolved $<2$ months) remission and 32.8\% for full (MDD resolved $>2$ months) remission of MDD (total: 79.7\%) using the PRIME-MD. Table 2 shows comparison between pre-and post-treatment outcomes using paired samples $t$-tests for the PHQ-9, psychosocial stressors from the BPHQ, the functional impairment item from the BPHQ, and the MAQ. This table also shows pre- and post-treatment reflections of Panic Disorder from the BPHQ. Results indicated broad and convergent findings suggesting improvements from pre- to post-treatment. Mirroring the BDI-II finding, mothers receiving IH-CBT reported drops in depressive symptoms as reflected by the PHQ-9. They also reported improvements in items reflecting stress, relationship difficulties, and social support. Although there was a 
reduction in mothers meeting criteria for Panic Disorder, this change was not statistically significant. In terms of the MAQ, mothers showed less dissatisfaction with motherhood from pre- to post-treatment $(\mathrm{M}=32.8(\mathrm{SD}=5.9)$ vs. $\mathrm{M}=29.0(\mathrm{SD}=4.5), t(63)=5.0, P<$. 001).

\section{Moderators of Depression and MAQ Outcomes}

In order to examine the possible influences of selected demographic and clinical variables on depression and MAQ outcomes, a series of repeated measures ANCOVAs were conducted on pre- and post-treatment BDI-II, PHQ-9, and MAQ, with these selected variables as factors and with pre-treatment values included as covariates. Selected variables were maternal age at enrollment determined by median split (younger mean $=19.46(\mathrm{SD}=$ $1.30)$ vs. older mean $=25.69(\mathrm{SD}=5.33)$ ), number of home visits received during course of treatment determined by median split $($ lower mean $=7.58(\mathrm{SD}=2.63)$ versus higher mean $=$ $16.35(\mathrm{SD}=4.44)$ ), low income (yes vs. no), maternal education (no high school degree versus high school degree or higher), marital status (married vs. unmarried), experienced violence in the past year (yes vs. no), panic disorder status at enrollment (yes vs. no), birth status at start of treatment (prenatal vs. postnatal), whether or not mothers were taking antidepressant medications at the start of treatment (yes vs. no), and home visitation model (HFA vs. NFP). Results indicated that that there were no significant interactions between these variables and pre-post outcomes $(P>.05)$.

\section{Discussion}

This study confirms and extends Ammerman et al. [13] by comparing mothers receiving IHCBT and home visitation with mothers in home visitation alone. After controlling for covariates, the Treatment Group showed a sizable reduction in symptoms relative to those in the Comparison Group. Moreover, at time 2 mothers receiving IH-CBT were more likely to have experienced significant improvement as indicated by a $250 \%$ reduction in scores on the BDI-II and meeting criteria for being asymptomatic based on self-reported depressive symptoms. To the extent that maternal depression is associated with poorer outcomes in home visitation and elevated risk for child maltreatment, these reductions support the use of IH-CBT as a feasible and effective treatment in the context of these prevention services.

Pre-post comparisons in the Treatment Group documented broad improvements in depression and related clinical features. The majority of treated mothers reported partial or full remission of MDD at post-treatment, reductions in depressive symptoms using the PHQ-9, decreased stress, and increased coping and social support from pre-treatment levels. Maternal stress, coping, and social support have all been linked to child abuse risk and are targets for change in home visitation programs. As a result, successfully treating depression has the potential to more broadly impact multiple areas of maternal functioning that are of interest to maternal and child health programs.

The clinical severity of depressed mothers in these home visitation samples is striking. Mean BDI-II scores for both the Treatment and Comparison Groups were high and consistent with diagnostic severity levels of moderate to severe depression [19]. Although the screening cutoff of $\geq 20$ on the BDI-II contributed to the selection of more severe cases, it is notable that home visitation populations attract a subgroup of mothers with clinically significant manifestations of depression [7]. Indeed, even though mothers in IH-CBT had a substantial drop in depression over the course of treatment, mean BDI-II scores at post-treatment were still relatively high. Although the number of new mothers reporting depressive symptoms tends to decrease from the perinatal period through 2 years postpartum [27], a sizable proportion of mothers (particularly in home visitation programs) continue to exhibit elevated levels over this interval. This is in part a reflection of the fact that most mothers in home 
visitation live in low resource and adverse environments which in turn contribute to vulnerability for continued depressive symptoms. It is possible that mothers may benefit from additional support after the end of treatment such as additional booster sessions. It was further found that rates of Panic Disorder, as determined by maternal self-report using the BPHQ, did not significantly change from pre- to post-treatment. This is likely attributable to the focused attention in IH-CBT directed towards the remediation of depressive symptoms and the secondary consideration given to other clinical concerns.

Mothers receiving IH-CBT reported increased satisfaction with motherhood and more positive views of children and child-rearing from pre- to post-treatment. This is noteworthy in that issues related to parenting and children were primary considerations of home visiting but ancillary to the depression treatment. The moderate correlation between negative maternal attitudes and depressive symptoms at post-treatment suggests that changes during treatment are linked to improvements in depression and are not an independent and secondary outcome of IH-CBT. This finding is consistent with the growing literature, conducted primarily with middle-class and lower risk populations of mothers, indicating that more positive views of children and parenting emerge with improvements in depression [28].

The finding that $19.9 \%$ the mothers in the Comparison Group received mental health treatment in the 9 month interval is consistent with other studies that have documented low levels of linkages with mental health treatment among those in home visitation [29]. Taking into account the reduced likelihood that these mothers obtained an evidence-based treatment, the high dropout rate in mental health services among low income populations, and the high non-adherence rate for depression treatments, it appears that depressed mothers in home visitation rarely receive adequate treatment in the community. Augmented treatments like IH-CBT provide a viable option to help a population that is well-known to mother and child health programs but faces considerable barriers to obtaining mental health treatments.

While home visitation is an opportune setting in which to identify and reach depressed mothers, some mothers were ineligible or did not complete treatment. In terms of eligibility, the majority of those who were not eligible for treatment either did not meet diagnostic criteria for MDD or had a comorbid psychiatric diagnosis incompatible with IH-CBT. Elevated depressive symptoms, therefore, are insufficient indicators of MDD and are often manifested in conjunction with other conditions that require different treatment strategies. A semi-structured psychiatric interview appears to be a useful adjunct to a self-reported screen to identify populations that are best served by an evidence-based treatment for depression. A sizable proportion of eligible mothers did not complete treatment, a finding that is highly consistent with observations of consumers of mental health services. Those who did not complete treatment were younger and less educated. Additional efforts are needed to engage and maintain these populations in treatment. These may include more sessions in the first weeks of treatment, further adaptations to IH-CBT to enhance commitment and motivation, or external incentives.

This study had several limitations that warrant caution in interpreting results. First, the open trial design does not permit definitive determination of treatment efficacy. A randomized clinical trial, in which participants are randomly assigned to treatment and control conditions, is needed to establish that IH-CBT is responsible for positive treatment outcomes. Second, not using an intent to treat strategy raises questions about outcomes for those mothers who began but did not complete IH-CBT. It is possible that the pre-post comparisons on completers only may yield inflated positive findings that would be diminished if the full sample of mothers were followed even if they dropped out of 
treatment. Third, there were several differences between the methods used to identify and assess the Treatment and Comparison Groups that might have influenced results. Some of these differences in method favor the Comparison Group. To the extent that mothers in the Comparison Group may not have had MDD, they comprise a less severe population in which improvement would be more likely. Also, mothers in the Comparison Group had 9 months to recover or experience a drop in symptom occurrence and severity, an interval that exceeds the length of a typical MDD episode [30], in contrast to the 4.5 months over which the Treatment Group was followed. Fourth, some of the outcomes in the pre-post comparisons were reflected by single items, and lengthier and more comprehensive measures of these constructs might yield different findings. Finally, although a semistructured clinical interview was used in the pre-post comparisons of mothers receiving IHCBT, this was not implemented with the comparison mothers.

The study also has several strengths. First, IH-CBT was developed a priori with adaptations to improve feasibility and impact based on setting, population, and context. Second, a multistep eligibility process was used for the Treatment Group to identify major depression, in contrast to much of the research with depressed mothers in home visitation in which recommended cutoffs of self-report measures are the sole means of identification [7]. Third, the sample sizes of 64 in the Treatment Group and 241 in the Comparison Group are large compared to what is typically reported in research on depressed mothers in home visitation. And fourth, IH-CBT is a structured and prescriptive treatment, lending itself to manualization and subsequent dissemination.

\section{Implications for Public Health}

The high prevalence and potential negative impacts of maternal depression in home visitation programs requires an effective and feasible response. IH-CBT has the potential to reach a large number of mothers who otherwise would not obtain treatment, alter both maternal lifecourse and child trajectories through reduction in symptoms and recovery from depression, and enhance the impact of a prevention approach that is widely implemented and has received a sizable public investment. The positive findings reported in this study warrant continued research on IH-CBT using more rigorous designs, more comprehensive assessment of mothers and children, and extended follow up to determine durability of gains.

\section{Acknowledgments}

Supported in part by Grant R34MH073867 from the National Institute of Mental Health. The authors acknowledge the participation and support of the United Way of Greater Cincinnati, Kentucky H. A. N. D. S., Ohio Help Me Grow, and www.OhioCanDo4Kids.org. We also thank Angelique R. Teeters for her helpful comments on the manuscript.

\section{References}

1. National Research Council and Institute of Medicine. Committee on Depression, Parenting Practices, and the Healthy Development of Children, Board on Children, Youth, and Families, Division on Behavioral and Social Sciences and Education. Washington, DC: The National Academies Press; 2009. Depression in parents, parenting, and children: Opportunities to improve identification, treatment, and prevention.

2. Lennon, M.; Blome, J.; English, K. Depression and low-income women: Challenges for TANF and welfare-to-work policies and programs. New York: National Center for Children in Poverty, Mailman School of Public Health, Columbia University; 2001.

3. Guterman, N. Stopping child maltreatment before it starts: Emerging horizons in early home visitation services. Thousand Oaks, CA: Sage; 2001. 
4. Kendrick D, Hewitt M, Dewey M, Elkan R, Blair M, Robinson J, et al. The effect of home visiting programmes on uptake of childhood immunization: A systematic review and meta-analysis. Journal of Public Health Medicine. 2000; 22:90-98. [PubMed: 10774910]

5. Sweet MA, Appelbaum MI. Is home visiting an effective strategy? A meta-analytic review of home visiting programs for families with young children. Child Development. 2004; 75:1435-1456. [PubMed: 15369524]

6. Astuto J, Allen L. Home visitation and young children: An approach worth investing in? Society for Research in Child Development, Social Policy Report. 2009; 23:3-22.

7. Ammerman RT, Putnam FW, Bosse NR, Teeters AR, Van Ginkel JB. Maternal depression in home visitation: A systematic review. Aggression and Violent Behavior. 2010; 15:191-200. [PubMed: 20401324]

8. Ammerman RT, Putnam FW, Altaye M, Chen L, Holleb LJ, Stevens J, et al. Changes in depressive symptoms in first time mothers in home visitation. Child Abuse \& Neglect. 2009; 33:127-138. [PubMed: 19328548]

9. Beck, AT.; Steer, RA.; Brown, GK. BDI-II manual. San Antonio: The Psychological Corporation; 1996.

10. Administration for Children and Families. Depression in the lives of early head start families [Brochure]. Washington, DC: Administration for Children and Families; 2006.

11. Duggan A, Fuddy L, Burrell L, Higman SM, McFarlane E, Windham A, et al. Randomized trial of a statewide home visiting program to prevent child abuse: Impact in reducing parental risk factors. Child Abuse \& Neglect. 2004; 28:623-643. [PubMed: 15193852]

12. DuMont K, Mitchell-Herzfeld S, Greene R, Lee E, Lowenfels A, Rodriguez M, et al. Healthy familes New York (HFNY) randomized trial: Effects on early child abuse and neglect. Child Abuse \& Neglect. 2008; 32:295-315. [PubMed: 18377991]

13. Ammerman RT, Putnam FW, Stevens J, Holleb LJ, Novak AL, Van Ginkel JB. In-home cognitive behavior therapy for depression: An adapted treatment for first-time mothers in home visitation. Best Practices in Mental Health. 2005; 1:1-14.

14. Beck, JS. Cognitive therapy: Basics and beyond. New York: Guilford Press; 1995.

15. Ammerman RT, Bodley AL, Putnam FW, Lopez WL, Holleb LJ, Stevens J, et al. In-home cognitive behavior therapy for a depressed mother in a home visitation program. Clinical Case Studies. 2007; 6:161-180.

16. Lau AS. Making the case for selective and directed cultural adaptations of evidence-based treatments: Examples from parent training. Clinical Psychology: Science and Practice. 2006; 13:295-310.

17. Olds DL. Prenatal and infancy home visiting by nurses: From randomized trials to community replication. Prevention Science. 2002; 3:153-172. [PubMed: 12387552]

18. Daro DA, Harding KA. Healthy families America: Using research to enhance practice. Future Child. 1999; 9:152-178. [PubMed: 10414015]

19. Steer R, Brown G, Beck A, Sanderson W. Mean BDI-II total scores by severity of major depressive episode. Psychological Reports. 2001; 88:1075-1076. [PubMed: 11597055]

20. Spitzer RL, Williams JB, Kroenke K, Linzer M, deGruy FV III, Hahn SR, et al. Utility of a new procedure for diagnosing mental disorders in primary care. The PRIME-MD 1000 study. JAMA. 1994; 272:1749-1756. [PubMed: 7966923]

21. Beck, AT.; Rush, AJ.; Shaw, FB.; Emery, G. Cognitive therapy of depression. New York: Guilford Press; 1979.

22. Blagys MD, Hilsenroth MJ. Distinctive activities of cognitive-behavioral therapy: A review of the comparative psychotherapy process literature. Clinical Psychologist. 2002; 22:671-706.

23. Keller MB. Past, present, and future directions for defining optimal treatment outcome in depression: Remission and beyond. JAMA. 2003; 289:3152-3160. [PubMed: 12813121]

24. Spitzer RL, Kroenke K, Williams JB. Validation and utility of a self-report version of PRIME-MD: The PHQ primary care study. JAMA. 2000; 282:1737-1744. [PubMed: 10568646]

25. Warner R, Appleby L, Whitton A, Faragher B. Attitudes toward motherhood in postnatal depression: Development of the Maternal Attitudes Questionnaire. Journal of Psychosomatic Research. 1997; 43:351-358. [PubMed: 9330234] 
26. Church NF, Brechman-Toussaint ML, Hine DW. Do dysfunctional cognitions mediate the relationship between risk factors and postnatal depression symptomatology? Journal of Affective Disorders. 2005; 87:65-72. [PubMed: 15922455]

27. Mayberry LJ, Horowitz JA, Declercq E. Depression symptom prevalence and demographic risk factors among U.S. women during the first 2 years postpartum. Journal of Obstetric, Gynecologic, and Neonatal Nursing. 2007; 36:542-549.

28. Gunlicks ML, Weissman MM. Change in child psychopathology with improvement in parental depression: A systematic review. Journal of American Academy of Child Adolescent Psychiatry. 2008; 47:379-389.

29. Administration on Children Youth and Families. Making a difference in the lives of children and families: The impacts of Early Head Start Programs on infants and toddlers and their families. Washington, DC: U.S. Department of Health and Human Services; 2002.

30. Kessler RC, Berglund P, Demler O, Jin R, Koretz D, Merikangas KR, et al. The epidemiology of major depressive disorder: Results from the National Comorbidity Survey Replication (NCS-R). JAMA. 2003; 289:3095-3105. [PubMed: 12813115] 
Table 1

Demographics and service characteristics of first time mothers in treatment $(\mathrm{N}=64)$ and comparison $(\mathrm{N}=$ 241) groups

\begin{tabular}{|c|c|c|c|c|}
\hline Variable & $\begin{array}{l}\text { Treatment group } \\
\text { M (SD) or N (\%) }\end{array}$ & $\begin{array}{l}\text { Comparison group } \\
\text { M (SD) or } \mathbf{N}(\%)\end{array}$ & $t$ or $x^{2}$ & $P$ value \\
\hline Mother's age & $22.57(4.96)$ & $20.15(4.18)$ & 3.94 & $<0.001$ \\
\hline Baseline BDI-II & $31.69(7.45)$ & $27.73(7.04)$ & 3.95 & $<0.001$ \\
\hline \multicolumn{5}{|l|}{ Race } \\
\hline Caucasian & $37(57.1 \%)$ & $171(70.8 \%)$ & 8.03 & $<0.05$ \\
\hline African American & $24(38.1 \%)$ & $67(27.9 \%)$ & & \\
\hline Latin American & $2(3.2 \%)$ & $3(1.3 \%)$ & & \\
\hline Native Hawaiian or other Pacific Islander & $1(1.6 \%)$ & $0(0.0 \%)$ & & \\
\hline \multicolumn{5}{|l|}{ Marital status } \\
\hline Single, never married & $60(93.7 \%)$ & $219(90.9 \%)$ & 0.85 & ns \\
\hline Married & $4(6.3 \%)$ & $20(8.3 \%)$ & & \\
\hline Divorced & $0(0.0 \%)$ & $2(0.8 \%)$ & & \\
\hline \multicolumn{5}{|l|}{ Education } \\
\hline Less than HS & $18(28.2 \%)$ & $123(50.9 \%)$ & 16.16 & $<0.01$ \\
\hline HS graduate or GED & $21(32.8 \%)$ & $76(31.6 \%)$ & & \\
\hline Partial college & $23(35.9 \%)$ & $38(15.8 \%)$ & & \\
\hline College graduate & $2(3.1 \%)$ & $4(1.8 \%)$ & & \\
\hline \multicolumn{5}{|l|}{ Home visitation model } \\
\hline HFA & $55(85.9 \%)$ & $198(82.2 \%)$ & 0.51 & ns \\
\hline NFP & $9(14.1 \%)$ & $43(17.8 \%)$ & & \\
\hline \multicolumn{5}{|l|}{ Low income } \\
\hline Yes & $57(89.1 \%)$ & $219(90.8 \%)$ & 0.00 & ns \\
\hline No & $7(10.9 \%)$ & $22(9.2 \%)$ & & \\
\hline
\end{tabular}


Table 2

Pre-treatment and post-treatment comparisons on MDD diagnosis, PHQ-9, BPHQ items, and MAQ for treatment group $(\mathrm{N}=64)$

\begin{tabular}{|c|c|c|c|c|}
\hline Variable & $\begin{array}{l}\text { Pre-treatment } \\
\text { M (SD) or N }(\%)\end{array}$ & $\begin{array}{l}\text { Post-treatment } \\
\text { M (SD) or N }(\%)\end{array}$ & $t$ or $\chi^{2}$ & $P$ value \\
\hline PHQ-9 & $15.05(4.50)$ & $6.05(5.12)$ & 10.89 & $<.01$ \\
\hline \multicolumn{5}{|l|}{ BPHQ (panic disorder) } \\
\hline PDO & $16(25.0 \%)$ & $9(14.1 \%)$ & 2.44 & ns \\
\hline No PDO & $48(75.0 \%)$ & $55(85.1 \%)$ & & \\
\hline $\begin{array}{l}\text { How difficult have these problems made it for you to do your work, take care of } \\
\text { things at home or get along with other people? }\end{array}$ & $2.60(0.83)$ & $1.70(0.61)$ & 6.79 & $<.001$ \\
\hline Worrying about your health & $2.08(0.80)$ & $1.92(0.82)$ & 1.26 & $\mathrm{~ns}$ \\
\hline Your weight or how you look & $2.44(0.66)$ & $2.23(0.77)$ & 1.94 & $<.10$ \\
\hline Little or no pleasure during sex & $1.95(0.87)$ & $1.63(0.79)$ & 2.57 & $<.05$ \\
\hline Difficulties with spouse, partner, or boyfriend & $2.31(0.78)$ & $1.79(0.81)$ & 3.75 & $<.01$ \\
\hline The stress of taking care of children, parents, or other family members & $2.11(0.81)$ & $1.73(0.72)$ & 3.10 & $<.01$ \\
\hline Stress of work outside of home or at school & $1.95(0.87)$ & $1.51(0.65)$ & 3.32 & $<.01$ \\
\hline Financial problems or worries & $2.65(0.65)$ & $2.10(0.76)$ & 4.93 & $<.01$ \\
\hline Having no one to turn to when you have a problem & $2.11(0.76)$ & $1.55(0.75)$ & 4.22 & $<.01$ \\
\hline Thinking or dreaming about something terrible that happened to you in the past & $2.02(0.88)$ & $1.34(0.65)$ & 5.12 & $<.01$ \\
\hline Something bad that happened recently & $2.08(0.85)$ & $1.70(0.82)$ & 2.65 & $<.01$ \\
\hline MAQ & $32.75(5.86)$ & $28.98(4.52)$ & 5.01 & $<.01$ \\
\hline
\end{tabular}

PHQ-9Patient Health Questionnaire-9, BPHQ Brief Patient Health Questionnaire, MAQ Maternal Attitudes Questionnaire 